\title{
Memoria profesional y Trabajo Social chileno. Reforma agraria y dictadura militar
}

\author{
Patricia Castañeda Meneses \\ Universidad de Valparaíso, Chile
}

\author{
Ana María Salamé Coulon \\ Universidad de La Frontera, Chile
}

\section{Memoria profesional y Trabajo Social chileno: reforma agraria y dictadura militar}

Resumen: El presente artículo se inscribe en el marco de un proyecto de investigación interuniversitario que contribuye al rescate de la memoria profesional del Trabajo Social chileno durante el período 1973-1990, en el contexto de la dictadura militar. En forma específica, se presenta un análisis integrado respecto de la participación de Trabajo Social en el proceso de Reforma Agraria implementado durante el período predictatorial 1960-1973, revisando las implicancias que tuvo para su desarrollo profesional, junto con analizar los devastadores efectos de la dictadura militar en la tarea especializada realizada en el agro chileno.

Palabras clave: Trabajo Social chileno. Dictadura militar. Memoria Profesional. Reforma Agraria.

\section{Memória profissional e Trabalho Social chileno: reforma agrária e ditadura militar}

Resumo: O presente artigo se inscreve no marco de um projeto de pesquisa interuniversitário que contribui ao resgate da memória profissional do Trabalho Social Chileno durante o período de 1973-1990, no contexto da ditadura militar. De maneira especifica se apresenta uma análise integrada sobre a participação do Trabalho Social no processo de Reforma Agraria organizado durante o período da pré-ditadura de 1960-1973, revisando as implicações que o mesmo teve para seu desenvolvimento profissional e junto com a análise dos efeitos devastadores da ditadura militar na tarefa especializada que foi realizada na agricultura chilena.

Palavras-chave: Trabalho Social chileno. Ditadura militar. Memória profissional. Reforma agrária.

\section{Professional Memory and Chilean Social Work: agrarian reform and the military dictatorship}

Abstract: This article is part of an interuniversity research project that contributes to the revival of the professional memory of Chilean social work from 1973-1990 in the context of the military dictatorship. In a specific form, it presents an integrated analysis of the participation of social work in the process of agrarian reform implemented during the period before the dictatorship, 1960-1973, reviewing the implications it had for its professional development, and also analyzes the devastating effects of the military dictatorship on the specialized task conducted in Chilean agriculture.

Keywords: Chilean Social work. Military dictatorship. Professional Memory. Agrarian Reform. 


\section{Presentación}

La Reforma Agraria se inicia en Chile a fines de 1962, bajo la administración del Presidente Jorge Alessandri Rodríguez. El proceso estuvo impulsado por los acuerdos de colaboración económica y de asistencia técnica internacional desarrollados desde Estados Unidos hacia los países latinoamericanos a través de la Alianza para el Progreso, iniciativa que buscaba evitar la multiplicación de la experiencia de la Revolución Cubana en el continente, a través de la promoción de reformas estructurales que enfrentaran las profundas desigualdades económicas y sociales que afectaban a América Latina. La Reforma Agraria contó con un fuerte respaldo de la Iglesia Católica chilena, organismos internacionales y partidos políticos progresistas. Los objetivos que perseguía este proceso, según Díaz (1972), eran los siguientes:

1- Redistribuir la propiedad agraria a través del mecanismo de expropiación, a fin de revertir la alta concentración que presentaba la tenencia de la tierra en Chile desde la época fundacional de la conquista española y que se expresaba en el complejo latifundio/minifundio. Según cifras del año 1955, se estimaba que los grandes propietarios agrarios concentraban el $65.4 \%$ de la tierra arable del país, mientras que los pequeños propietarios poseían el 12.6\% del recurso (SUÁREZ, 1972);

2- Incorporar a la propiedad de la tierra a las familias campesinas;

3- Mejorar la situación productiva agraria nacional; y,

4- Realizar una promoción del sector rural a fin de integrarlo activamente a la vida nacional.

Entre 1962 y 1964 la Reforma Agraria se aplicó a través de la Ley № 15.020, conocida como "la reforma del macetero" por su escasa operatividad, ya que definía que el mecanismo de expropiación aplicaba solamente en los predios mal explotados o evidentemente abandonados. Asimismo, el cuerpo legal establecía que el precio del predio a expropiar debía ser discutido en tribunales y su pago efectuarse al contado, dilatando los tiempos de toma de posesión de la tierra por parte de los organismos estatales y estrechando la disponibilidad de los presupuestos públicos destinados al agro (DÍAZ, 1972).

Durante el gobierno de Eduardo Frei Montalva (1964-1970), se aceleró apreciablemente el proceso respecto de la etapa anterior, ya que hasta 1967 se aplicó con mayor flexibilidad la normativa vigente y se desarrolló complementariamente un nuevo proyecto de ley que consideraba un sistema de pagos diferido y ampliaba las expropiaciones a las propiedades agrícolas que superaban las 80 hectáreas de riego básico (H.R.B.), medida que permitía establecer las equivalencias del potencial productivo entre suelos de diferentes calidades y ubicaciones, tomando como referente la capacidad de producción de una hectárea física del Valle del Maipo, considerada como la mejor tierra agrícola del país en aquellos años. El 28 de julio de 1967 se promulga la nueva Ley de Reforma Agraria $\mathrm{N}^{\circ} 16.640$, que pasa a reglamentar los predios que ya habían iniciado su proceso con el anterior cuerpo legal y en aquellos que se incorporaron con posterioridad. En forma convergente, se estimuló la sindicalización campesina a través de la Ley No 16.625 del 29 de abril de 1967, reconociendo este derecho social en inquilinos, afuerinos, voluntarios y minifundistas, lo que generó una importante e inédita convocatoria sindical que incrementó progresivamente su cobertura desde 76.356 campesinos en 1969 hasta 277.895 en 1972 (ARMIJO; CAVIEDES, 1997). Entre los años 1970 y 1973, durante el gobierno del Presidente Salvador Allende Gossens, la Reforma Agraria se profundizó a través de la relativización de las causales de expropiación consideradas formalmente en la ley, impulsando ocupaciones de hecho de los predios, acciones que fueron conocidas como "tomas de fundo" (GIA, 1979, p. 33), las que aumentaron de 9 episodios en 1967 a 1.278 ocupaciones en 1971 (ARMIJO; CAVIEDES, 1997).

La normativa legal establecía que una vez expropiado el predio, las familias campesinas que allí vivían y querían voluntariamente permanecer en el mismo, pasaban a constituir un asentamiento. Esta nueva organización mantenía la estructura de trabajo agraria previa a la expropiación, a fin de minimizar efectos productivos negativos generados por el cambio de gestión económica realizado. La etapa de asentamiento permitía que la mayoría de las decisiones internas de la explotación fueran tomadas por la propia organización campesina con apoyo de los organismos estatales que asesoraban las decisiones de planificación, inversión, crédito, comercialización y abastecimiento de la producción agrícola. Una vez concluida la etapa de asentamiento, estimada inicialmente en tres años de duración, las familias campesinas podían optar a formas de asignación de tierras, en modalidades de propiedad exclusiva, propiedad compartida a través de cooperativas o propiedad exclusiva con copropiedad de bienes comunes (MATTELARD, 1970).

Como resultado de las medidas de cambio estructural asumidas, el sector agrario reformado llegó a representar el $40.5 \%$ de la superficie regada del país y el $28.1 \%$ de la superficie agrícola del país, estimándose en más de 76 mil las familias campesinas beneficiadas con el proceso (SUÁREZ, 1972). Asimismo, la implementación de la Reforma Agraria generó una institucionalidad estatal en base a la redefinición de tareas 
de los organismos existentes y la creación de servicios especializados. La red institucional estuvo compuesta por la Corporación de Reforma Agraria (CORA), la Oficina de Planificación Agrícola (ODEPA), el Instituto de Desarrollo Agropecuario (INDAP), el Servicio Agrícola y Ganadero (SAG), la Dirección General de Aguas y la Empresa Nacional de Riego.

En este marco histórico social, el trabajo social se incorporó en las importantes tareas y desafíos que representaba el proceso de cambio agrario estructural para el campesinado chileno y desarrolló innovadoras experiencias profesionales que forman parte de su memoria histórica profesional en el período predictatorial. Así entonces, el presente artículo expone las principales características que asumen los procesos de formación en trabajo social y el desempeño profesional en el marco de la Reforma Agraria y las consecuencias profesionales derivadas del traumático cambio en las condiciones políticas del país a contar de Septiembre de 1973.

\section{Metodología}

El presente artículo es resultado de una investigación cualitativa que busca el reconocimiento y valorización de la memoria colectiva profesional del trabajo social chileno en el contexto de la dictadura militar (CASTAÑEDA; SALAMÉ, 2013). El objetivo específico analizado en esta oportunidad, corresponde a la reconfiguración del periodo profesional predictatorial en el marco de las transformaciones estructurales generadas por la Reforma Agraria en el mundo rural chileno y su influencia en la formación y desempeño profesional de trabajo social. Las estrategias de recolección de información corresponden a entrevistas biográficas aplicadas a cinco estudiantes y seis profesionales diferenciados conforme el rol desempeñado por cada sujeto durante el periodo en estudio (1960 a 1973) y todos ellos vinculados a experiencias en distintos sectores agrícolas de la zona centro y sur del país. También se recurrió a la revisión documental de archivos de escuelas universitarias tradicionales, tesis o trabajos de título, cuerpos legales vinculados a la profesión y publicaciones profesionales de la época. Finalmente, el plan de análisis organiza la información conforme a criterios de relevancia, significancia y convergencia aportados desde los propios protagonistas y las fuentes secundarias consultadas. Se mantiene el lenguaje sexista sin correcciones, al ser referido directamente al contexto y a los documentos de la época ${ }^{1}$.

\section{Resultados: Trabajo Social y Reforma Agraria}

Durante el período 1963-1970, la Corporación de Reforma Agraria (CORA) consolidó una planta de 21 trabajadoras sociales titulares y respaldó un total de 145 experiencias formativas de estudiantes en prácticas profesionales de diversas universidades. Las experiencias de trabajo social en el medio agrario, recopiladas a través de tareas de supervisión técnica en terreno, permitían reconocer dos etapas de actuación de trabajo social en el proceso. Según Garlaschi (1970), en la primera etapa, situada entre 1963 a 1965, las acciones se relacionaron preferentemente con la selección y organización de los asignatarios de tierras, pertenecientes a colonias agrícolas de las provincias de Santiago, O’Higgins, Cautín, Arauco y Osorno, en el marco de la Ley No 15.020. La segunda etapa, entre 1965 y 1970 se caracterizó por un intenso trabajo de alfabetización campesina y de organización y preparación para las diferentes etapas que va cumpliendo la Reforma Agraria. La labor se proyectó ampliamente para las escuelas de servicio social de casi todo el país.

Los programas en que Servicio Social de la CORA participó en su programación y realización eran: Alfabetización y Educación Básica a nivel zonal, área y asentamiento; Capacitación Organizacional a partir de las orientaciones de la Ley 16.640 referidas a asentamiento, asamblea, consejos de administración, comités ejecutivos y otras organizaciones de base; Asignación de tierras en relación a tipo de propiedad y aspectos técnicos y Organización Cooperativa; y Coordinación y Capacitación Institucional a nivel campesino y funcionario centrado en aspectos de seguridad social y previsión (GARLASCHI, 1970).

Asimismo, dado que a contar del año 1962 las escuelas de Servicio Social de la Universidad de Chile, de las sedes Valparaíso y Temuco habían comenzado a aplicar el método de Organización y Desarrollo de la Comunidad en zonas urbanas, su aplicación pudo ampliarse a partir del año 1964 en comunidades rurales, bajo el patrocinio del Instituto de Educación Rural y, a contar de 1965 en predios reformados, con el respaldo institucional de la CORA (FIGUEROA, 1976). En los años siguientes, la CORA ampliaría su patrocinio institucional a través de convenios de colaboración con las escuelas de Servicio Social de la Universidad del Norte (Arica); Universidad de Chile, sedes La Serena y Santiago; Universidad Católica de Valparaíso, Universidad Católica de Chile, Universidad de Concepción y Dr. Alejandro del Río de Santiago. Los objetivos de los convenios eran "incorporar al alumno de cuarto año y al egresado memorista a la realidad social agraria y lograr la creación y recreación de metodologías acordes con el planteamiento del proceso agrario" 
(GARLASCHI, 1970, p. 42). En este marco de colaboración, las acciones profesionales de Trabajo Social se desarrollaban en torno a tres líneas centrales de trabajo, las que correspondían a alfabetización, organización campesina y cooperativismo.

\section{Trabajo Social, Reforma Agraria y Alfabetización Campesina}

En Chile a inicios de la década de 1960, la tasa de analfabetismo a nivel nacional alcanzaba el 16,4\%. La cifra se elevaba a $33.6 \%$ en la población rural, situación que impedía la plena incorporación del campesinado a la comunidad nacional (ARMIJO; CAVIEDES, 1997). Por tanto, la Reforma Agraria impulsó procesos de alfabetización y educación básica, considerados como "la primera herramienta para la participación activa del campesino en la vida nacional" (CORA, 1970, p. 55).

Las estrategias de alfabetización campesina se apoyaban metodológicamente en los planteamientos de pedagogía crítica aportados por la experiencia de Paulo Freire en Brasil. La tarea educativa de trabajo social valoraba el principio central de la educación popular, respecto que la lectura del mundo precede a la lectura de la palabra y los planteamientos pedagógicos expuestos en las obras La Educación como práctica de la libertad (1967) y Pedagogía del Oprimido (1968) respaldaban el aporte profesional en los cambios estructurales requeridos en el agro. En este sentido, la labor educativa se enmarcaba en la convicción de que el fin del latifundio no significaba el fin de las relaciones sociales que existían en su interior, por lo era necesario impulsar una transformación profunda de la "cultura del silencio" de aquellos campesinos que ahora debían hacerse cargo de sus labores productivas (CORA, 1968, p. 27):

Yo salí a trabajar alfabetizando a los campesinos. Imagínense ustedes las manos de un hombre de campo, son gruesas, toscas. Y esas manos gorditas no sabían cómo tomar un lápiz, eso cuesta mucho, mucha educación y es algo tan simple para nosotros, tan rutinario para nosotros. Entonces le enseñabas que no es un ignorante porque no sabe escribir. El carece de la capacidad de escritura. Tú como profesional lo estabas preparando, pero uno aprendía de la vida y se enriquecía con la experiencia del campesino. O sea era un proceso de entrega mutua. Y lo más maravilloso fue cuando aprendieron a firmar (Estudiante Trabajo Social, Universidad de Chile, Santiago. Período 1964-1968).

Los esfuerzos en alfabetización campesina realizados por trabajo social se enmarcaban en las orientaciones reformistas, que buscaban proporcionar al campesino beneficiario del proceso los contenidos instrumentales necesarios que le permitan tener "una base fundamental de conocimientos para lograr incorporarse mejor a su capacitación técnico empresarial y organizacional" (CORA, 1968, p. 23).

Los aprendizajes profesionales obtenidos en las experiencias de alfabetización adulta rural realizadas en la época, indican la existencia de una relación virtuosa entre alfabetización y organización campesina, siendo frecuente que estas iniciativas educativas fueran apoyadas por la motivación de los dirigentes y por un compromiso de aprendizaje expresado frente a toda la asamblea:

Pero el pilar de toda la motivación, confiesa ella, fue el presidente del asentamiento, un hombre semianalfabeto quien con las propias frases de sus compañeros los entusiasmaba a estudiar (...). En la segunda etapa, el asentamiento como organización de los campesinos se hizo cargo del problema, el cual fue tratado en Asamblea (...) Realizada la encuesta, la tabulación se hizo en la Asamblea (...) Comprometidos ante el asentamiento, éste dio facilidades, pero también sanciones. A cada uno de los alumnos se les computó el tiempo de clases como tiempo trabajado y a aquellos que faltaban se les bajó las calificaciones, punto importante en la postulación como asignatario definitivo de la tierra (...) Los nuevos alumnos pudieron elegir sus propios profesores, aquellas personas de la comunidad a las cuáles respetaban y tenían confianza (...) Los profesores tuvieron capacitación especial con el método de Paulo Freire. A través de ella, que abarcaba una parte filosófica, otra sindical y otra técnica. Se pretendía que ellos hicieran "hablar las letras". Según testimonios de los propios alumnos, las letras hablaron de aquello que más importante les parecía a ellos (...) Hablaron en los conceptos familiares de arado, cosecha, compañero, patrón, etc., fueron las letras hilvanadas que hablaron (...) Esta era una tarea del asentamiento, por eso cada 15 días se evaluaba con la asistente social y los profesores (De la entrevista realizada por Luz María Prado a Juanita Aravena, Trabajadora Social de CORA, 1970, p. 44-45).

La estrategia pedagógica de trabajar con conceptos reconocibles desde la cotidianeidad campesina, generaba mapas culturales que favorecían los vínculos entre pedagogía y concientización, facilitando la lectura 
crítica y transformadora de la realidad. En 1970, se estimaba que los programas de alfabetización implementados por CORA en el período 1965-1970 y en los que trabajo social participaba activamente, habían beneficiado a un total de 35 mil campesinos (ICIRA; 1970, p. 9)

\section{Trabajo Social, Reforma Agraria y Organización Campesina}

El cumplimiento del objetivo de la Reforma Agraria relacionado con el aumento de la producción agrícola, exigió una labor de capacitación orientada a posicionar al campesinado como sujeto central en el proceso de transformación estructural de la agricultura. Por tanto, se realizó un trabajo profesional destinado a impulsar un cambio profundo en los valores campesinos tradicionales "arraigados en una relación de dependencia y subordinación respecto de la naturaleza, del sistema de trabajo y del sistema social al que pertenecía" (CORA, 1970, p. 55). Por consiguiente, la intervención social se orientó a la formación y fortalecimiento de la organización y participación campesina, para respaldar la toma de decisiones colectivas en los ámbitos productivos, organizacionales y comunitarios. "Romper las barreras entre campesinos, creadas por muchos años de aislamiento. Entender que en la medida que fueran solidarios en la gestión económica que iban a realizar unidos, habría mayor probabilidad de incrementar los beneficios obtenidos" (CORA, 1970, p. 55).

Las experiencias formativas de las estadas prácticas de trabajo social se definieron desde el acompañamiento organizacional en los procesos productivos campesinos, en el marco del compromiso ideológico explícito que se declaraba respecto de las transformaciones estructurales en curso:

En el año 1972 yo me encontraba haciendo mi práctica integrada en el asentamiento Roble Huacho, en el marco del convenio de la Universidad de Chile con la CORA. El fundo había sido expropiado y se habían traído familias de distintos lugares del país para ocupar el asentamiento. La práctica consistía en incorporarse al asentamiento como uno más. Por eso, realizábamos las tareas agrícolas junto con los campesinos, codo a codo con ellos. En ese tiempo tuvimos que ralear remolacha, así buscábamos incorporarnos a las tareas cotidianas que desarrollaban los campesinos. Eso era parte de la estrategia de entrada, para luego realizar las funciones y tareas que teníamos asignadas. Esas tareas eran ayudar a la organización del asentamiento. En forma paralela había un trabajo ideológico importante, consistía en remarcar que este era un gobierno de izquierda, elegido por el pueblo y para el pueblo. Así, los componentes de la práctica eran tres: político, organizacional y productivo. (Estudiante en Práctica Profesional de Trabajo Social. Universidad de Chile, sede Temuco, 1972).

En ese marco, la participación campesina era promovida técnica e ideológicamente por trabajo social en tres niveles: a nivel de asentamiento, donde el campesinado local era ejecutor responsable de la producción agropecuaria; a nivel de zonas provinciales y regionales a través de Federaciones Campesinas que participaban en la toma de decisiones de acción organizacional y de responsabilidad financiera; $y$, a nivel central, a través de la Confederación Nacional, en donde se discutían los fundamentos y proyecciones del proceso reformista y las políticas generales de implementación (CORA, 1970, p. 60)

En 1970, se estimaba que en el período 1966-1970 se habían capacitado 28.402 campesinos en los programas de capacitación organizacional y sindical campesina en los que participaban profesionales de trabajo social, de los cuáles 8.783 poseían la calidad de dirigentes (ICIRA; 1970, p. 9).

\section{Trabajo Social, Reforma Agraria y Cooperativismo Campesino}

Durante la implementación de la Reforma Agraria, la organización cooperativa campesina fue concebida como una forma de trabajo que permitía emplear todos los recursos de suelo, maquinaria, mano de obra e insumos disponibles, aumentando las ganancias por economía a escala y por comercialización de mayores volúmenes de productos. El proceso productivo agrario de los predios reformados se definió en términos de metas de producción y, por sobre todo, en su aspiración de integración del campesinado a la economía nacional, respaldando su participación en las estructuras de poder y en el cumplimiento de su rol en el desarrollo del país (CORA, 1970, p. 83). Se analizó críticamente la estructura agraria tradicional derivada del complejo latifundio/ minifundio y se definió como técnicamente necesario "fomentar las asignaciones de tipo comunitario, las que hasta el momento, se han efectuado en un 90\%" del total de los predios reformados (CORA, 1970, p. 45). Los fundamentos relacionados con esta medida indicaban que el sistema cooperativo permitiría grandes economías en el mejor manejo de la maquinaria, la utilización racional de las aguas, las empastadas y en general, de todos 
aquellos bienes de infraestructura indirectamente productivos como galpones, sitios, corrales y otros recursos. En esta tarea, trabajo social aportaba en forma central, en la medida que desarrollaba acciones formativas, orientadas a generar capacidades en la comunidad campesina para decidir libremente por el común acuerdo de sus miembros "la forma de explotar su tierra, ya sea determinando que cada campesino trabaje una parte o cultivando paños en común” (CORA, 1970, p. 45).

Para fortalecer el trabajo cooperativo, el colectivo profesional realizó un esfuerzo de integración que incluía a las mujeres, a fin de sumarlas a los procesos productivos cooperativos desde sus propias faenas agrícolas distintivas:

\begin{abstract}
Teníamos reuniones con los hombres y con ellos trabajábamos en capacitación, apoyando a que conocieran las distintas funciones de cada uno en los cargos de la directiva. También haciendo sensibilización para que las mujeres se incorporaran a la organización y en la directiva. La primera reunión en que participaron las mujeres del asentamiento fue bien tensa. Todos los hombres a un lado y todas las mujeres atrás en la sala. Los hombres las miraban raro como diciendo ¿Qué hacen aquí? El trabajo con las mujeres consistía en animarlas para que participaran en la organización. Y por otra parte se las organizó para que se incorporaran a la producción: fabricación de quesos y venta de huevos. Los dos productos se comercializaban en el mercado municipal de Temuco (Estudiante en Práctica Profesional de Trabajo Social, Universidad de Chile, sede Temuco, 1972).
\end{abstract}

A modo de ilustración de estas experiencias formativas cuyo foco de atención son las mujeres, puede mencionarse la memoria de titulación denominada "Productividad de la mujer en el Asentamiento Alameda" realizada en la sede Temuco de la Universidad de Chile (CASTILLO; CONCHA; SANDOVAL; TRUJILLO, 1972) cuyos objetivos se orientaban a medir la incorporación activa de la mujer campesina en las actividades económicas agrícolas, amplificando a través de la intervención social especializada, los aportes en asistencia técnica productiva que realizaba la Corporación de Reforma Agraria (CORA) y el Servicio Agrícola y Ganadero $(\mathrm{SAG})$ en las organizaciones rurales femeninas.

\title{
Trabajo Social, Golpe de Estado y Contrarreforma Agraria
}

A partir del Golpe Militar, el proceso de expropiaciones derivado de la Reforma Agraria se paralizó abruptamente y se reprimió la actividad organizacional y cooperativa campesina.

En el gobierno militar murieron muchos, que lo único que hacían era trabajar la tierra. Nosotros estuvimos capacitando en Caimanes, gente muy buena. Yo preguntaba por un campesino, me respondían lo mataron, lo fusilaron, Otro, está desaparecido. Eso fue muy duro, muy triste. Esa gente que yo compartía en sus casas, gente muy cariñosa, compañeros de trabajo que murieron (Trabajadora Social, funcionaria CORA, Período 1972-1973).

Según los antecedentes recopilados por la Vicaría de la Solidaridad, en el agro chileno fueron torturados o asesinados cientos de trabajadores rurales pertenecientes a organizaciones campesinas y sindicatos de antiguos latifundios, que fueron detenidos en los días posteriores al Golpe de Estado por patrullas militares o civiles armados. Una vez detenidos, eran ejecutados a orillas de ríos o en los puentes y desde allí lanzados para que fueran arrastrados por las aguas. Algunos de ellos permanecen como detenidos-desaparecidos a contar de la fecha de su detención. Asimismo, funcionarios de CORA y de reparticiones públicas ligadas al agro, fueron acusados de fomentar el activismo político y también fueron víctimas de acciones de represión por parte de las autoridades golpistas. Los operativos militares, allanamientos, arrestos arbitrarios y amedrentamientos en los predios reformados fueron una constante en los primeros años de instauración de la dictadura (DAIRE, 1992).

Frente al proceso de Reforma Agraria, las autoridades militares modificaron las condiciones estructurales de su implementación y desestimaron su futura contribución al desarrollo nacional. A partir de septiembre de 1973, la CORA se limitó a regularizar la situación de los predios expropiados a la fecha, bajo la figura de la Oficina de Normalización Agraria ODENA. Las plazas profesionales de trabajo social fueron suprimidas, suspendiéndose todo tipo de iniciativas relacionadas con tareas alfabetizadoras, organizativas y cooperativas. En diciembre de 1973 se dictó el Decreto Ley 208 que prohibió asignar tierras a aquellos dirigentes o campesinos que hubiesen participado de ocupaciones de hecho o "tomas de fundos". La Corporación de Reforma Agraria como repartición pública fue disuelta definitivamente como institución en el año 1979 y la Ley de Reforma Agraria No 16.640 fue derogada en 1989, durante los meses finales de la dictadura militar: 
Los militares pensaban que los trabajadores sociales eran activistas políticos, porque no entendieron que los trabajadores sociales estábamos trabajando con los campesinos, en los sindicatos, en las comunidades, alfabetizando, organizando. Todo esto, ellos lo consideraron subversivo. (Trabajadora Social, funcionaria CORA, Período 1972-1973).

En los años posteriores al Golpe Militar se configuró un proceso conocido en el mundo rural como la Contrarreforma Agraria, en donde la producción agropecuaria se orientó a un modelo agro exportador, en condiciones económicas de libre mercado e inexpropiabilidad de la tierra agrícola. En el marco de este proceso, se estimaba que a 1979 el 29\% de la superficie total expropiada entre los años 1962 y 1973 había sido devuelta a sus antiguos propietarios; el 39\% de la superficie había sido considerada no apta para producción agrícola y se licitó a grupos financieros o antiguos propietarios expropiados; y el 33\% de la tierra expropiada fue asignada a familias campesinas, sin asistencia técnica estatal ni acceso preferente al crédito agrícola. De las 38 mil familias campesinas asignadas, sólo 20 mil pudieron conservarla en los primeros años. Posteriormente, la propiedad campesina se fue reduciendo hasta niveles críticos, y los predios reformados ingresaron al mercado de tierras, reconvirtiendo sus nuevos propietarios los procesos productivos agrícolas, ganaderos y forestales, conforme las reglas de oferta y demanda vigentes a nivel nacional e internacional (COX, 1982, p. 118).

\section{Discusión}

Con la Reforma Agraria, el sector rural chileno modifica estructuralmente sus condiciones de acceso y distribución de la propiedad agrícola. El complejo latifundio/minifundio, que constituía el histórico eje ordenador de la producción agrícola en Chile, da paso a la empresa agroexportadora generando profundos cambios en las concepciones que se tenían sobre la tierra, el tipo de producción agrícola, el mercado de destino de la producción y el rol del campesinado en el proceso productivo. En ese marco, y a pesar de su traumático final, la experiencia reformista representó para trabajo social oportunidades de desarrollo profesional que amplificaron su tradicional tarea asistencial, validando la promoción social como aprendizaje profesional del período, expresado principalmente a través de la alfabetización de adultos y del acompañamiento a la organización y producción cooperativa campesina. La dinámica reformista del agro contribuyó a impulsar la revisión del quehacer profesional en torno a los procesos de reconceptualización que caracterizaron el período a nivel latinoamericano, consolidando definitivamente la Organización y Desarrollo Comunitario como ámbito de intervención distintivo y especializado, junto con la redefinición desde su rol profesional clásico como garante de la estabilidad social hacia una renovada propuesta de agente de cambio social.

Los trabajos de titulación de trabajo social desarrollados en la Universidad de Chile, en el marco del proceso reformista, sistematizaban experiencias de investigación, intervención y reflexión profesionales que ilustran estas redefiniciones y cuestionamientos: "El comunero de Potrerillos frente al proceso de Reforma Agraria" (1968); "Estudio de actitudes de los campesinos del valle del Choapa frente a la erradicación" (1969); "Algunos problemas que se generan en el proceso de Reforma Agraria 1.- Situación Laboral y 2.- Enfrentamiento del campesino con la nueva estructura" (1970); "Estudio cooperativo de los resultados obtenidos con la aplicación del método psicosocial de Paulo Freire en asentamientos del área San Felipe III Zona de Reforma Agraria" (1972); "Hacia una praxis de servicio social en reforma agraria en un período de transición al socialismo" (1972); "La toma de fundo como una expresión de la conciencia campesina" (1973). Las experiencias identificadas impulsaban procesos de transformación agraria a través de objetivos de trabajo que se orientaban a "Conocer las principales problemas sociales que afectan al Asentamiento campesino" realizando esfuerzos profesionales "con el objeto que en base a resultados, se puedan formular planes para un proceso de cambio social” (SANDOVAL; CASTILLO; TRUJILLO; CONCHA, 1972, p. 2-3).

La contingente situación política, económica y social de la época impulsó al trabajo social chileno a cuestionar su quehacer y a imponerse nuevos desafíos profesionales que le permitieran ser parte activa de las acciones reformistas impulsadas desde el Estado:

El trabajador social se enfrenta a una problemática dura y difícil. Cualquiera que sea su posición debe saber que no puede contribuir a esconder los vicios del sistema: al contrario, debe entregar toda su técnica, todos sus conocimientos al campesino, para que sabiéndolo todo, juzgándolo todo, pueda actuarlo todo [...] Es el trabajador social el que debe capacitar para que el campesino participe a nivel de la planificación, de la programación, entregándole las técnicas necesarias para desenvolverse en un medio nuevo, con conciencia de clase, integrado totalmente a la base. Creo que el trabajador social deberá luchar en cualquier sistema político para que la clase explotada participe realmente del poder, su responsabilidad es investigar, crear y 
recrear metodologías de capacitación para hacer posible la construcción de una sociedad más justa y más humana (Garlaschi, 1970, p. 43).

Este despliegue de transformación profesional se detiene traumáticamente con el Golpe de Estado de septiembre de 1973, desencadenando una brutal acción represiva inicial, que dio paso a una Contrarreforma Agraria que desconoció los avances de la redistribución de la propiedad de la tierra y las redefiniciones cooperativas, comunitarias y de iniciativa individual campesina en torno a la producción, el capital y el trabajo agrícola impulsadas hasta ese aciago momento. El violento final del proceso reformista vio también extinguirse las experiencias formativas y profesionales de trabajo social en el mundo rural reformado.

En los adversos años venideros, la profesión debió sobrevivir censurada en la academia y cuestionada en su desempeño laboral, debiendo adscribirse forzosamente a una fuerte impronta tecnocrática que invisibilizó

\section{El complejo latifundio/}

minifundio, que constituía el

histórico eje ordenador de la

producción agrícola en Chile,

da paso a la empresa

agroexportadora generando

profundos cambios en las

concepciones que se tenían

sobre la tierra, el tipo de

producción agrícola, el

mercado de destino de la

producción y el rol del

campesinado en el proceso

productivo. progresivamente las particularidades del mundo rural en su trabajo profesional. Con ello, se bloquearon además las posibilidades de análisis y de reflexión de las transformaciones económicas, sociales y culturales que asumían los procesos de modernización del agro, precipitados por la aplicación del modelo de libre mercado en las zonas productivas reformadas. Sólo los acotados esfuerzos desarrollados a lo largo de 17 años de dictadura militar en las organizaciones no gubernamentales del mundo rural, permitieron que la forzada omisión profesional no se transformara en olvido total ni en abandono permanente. A pesar de lo anterior, la memoria profesional del período 1960-1973 cautela su condición de etapa pródiga en compromiso social y rescata para las generaciones venideras la definición de agentes de cambio, como síntesis del esfuerzo desplegado en la búsqueda de transformaciones estructurales que entregaban oportunidades de futuro a las precarias condiciones ancestrales de vida y de trabajo campesinas.

Hoy en día, los vestigios de la participación de trabajo social en la Reforma Agraria son casi inexistentes, dado que los textos, publicaciones y registros profesionales fueron eliminados por la censura, la represión y la persecución política ideológica que aplicó la dictadura a contar de septiembre de 1973 en las aulas universitarias y en los organismos y servicios públicos relacionados con el proceso reformista. Por tanto, los testimonios de quienes fueron testigos directos de la época y los escasos textos que no fueron destruidos o permanecieron olvidados en los anaqueles de las bibliotecas, son los únicos retazos de la memoria que han evitado la pérdida total del relato histórico de trabajo social en la Reforma Agraria y permiten resignificar a la profesión que acompañó las esperanzas campesinas de superar la cultura del silencio a través de la palabra escrita, la organización social y el esfuerzo cooperativo; y compartió su profundo dolor por no alcanzar a ver el despliegue de un anhelado nuevo amanecer para el agro chileno.

\section{Referencias}

ARMIJO, G. Y; CAVIEDES, B. Vicisitudes y Cambios en el Mundo Rural Chileno. La última modernización agraria ¿La gran solución de fin de siglo? En: Anales de la Universidad de Chile, Sexta Serie n.5, octubre de 1997. Disponible en: <http://www.analesuchile.cl/6s/ n5/estudios1.htm>. Visitada en 24 set. 2014.

CASTAÑEDA, P.; SALAMÉ, A. M. Trabajo Social chileno y Gobierno Militar. 40 años de memoria y olvido. Revista de Trabajo Social. Santiago de Chile: Pontificia Universidad Católica de Chile, n. 84, p. 55-66, 2013.

CASTILlO, D.; CONCHA, M.; SANDOVAL, A. y TRUJILlO, A. Productividad de la Mujer en el asentamiento Alameda. Universidad de Chile: Temuco, 1972.

CORA (CORPORACION DE REFORMAAGRARIA). Informe del Programa de Educación Básica. Santiago de Chile: Departamento de Desarrollo Campesino Central, 1968. 
Reforma Agraria Chilena 1965-1970. Santiago de Chile, 1970.

COX, M. Artículo: La Pequeña Agricultura Chilena: Condiciones Actuales y Perspectivas. En: Agricultura Chilena 1974-1982: Políticas, Evolución y Campesinado. Santiago de Chile: Desarrollo Campesino DECAM S.A., 1982.

DAIRE, A. El proceso represivo: Documento de Trabajo. Vicaría de la Solidaridad: Santiago de Chile, 1992. Disponible en: <http:// www.archivovicaria.cl/archivos/VS4b4ca19daa347_12012010_121pm.pdf>. Visitado en: 21 nov. 2014.

DÍAZ, L. La propiedad en la Ley de Reforma Agraria. Santiago de Chile: Editorial Andrés Bello, 1972.

FIGUEROA A. Evolución del servicio social profesional en Chile, durante el período comprendido entre los años 1925 y 1975. Universidad de Chile, sede Valparaíso, 1976.

FREIRE, P. La educación como práctica de la libertad. Tradução de Loretta Slover. Santiago: Instituto de Capacitación e Investigación en Reforma Agraria ICIRA, 1969.

GARLASCHI, A. Trabajo Social en la Reforma Agraria. En: Revista de Trabajo Social, Pontificia Universidad Católica de Chile. Revista de Trabajo Social n.1. Santiago de Chile. p. 41-43, 1970.

GIA (GRUPO DE INVESTIGACIONES AGRARIAS) Parceleros de la Reforma Agraria. En: Cuadernillo de Informaciones Agrarias n.2. Santiago de Chile, 1979.

GÓMEZ, L. El Asentamiento Campesino Chileno. Apuntes del Seminario Rural Escuela de Trabajo Social. Revista de trabajo social, n.1. Santiago de Chile: Pontificia Universidad Católica de Chile. p. 30-34, 1970.

ICIRA. Síntesis de algunos aspectos de la Reforma Agraria Chilena 1964-1969. Instituto de Capacitación e Investigación en Reforma Agraria ICIRA. Santiago de Chile, 1970.

MATTELARD, A. Apuntes sobre el concepto de reversibilidad de un proceso de Reforma Agraria. En: Reforma Agraria Chilena. Seis Ensayos de interpretación. Santiago de Chile: Instituto de Capacitación e Investigación en Reforma Agraria, 1970.

PRADO, L. Campesinos hicieron hablar las letras. De la entrevista realizada por Luz María Prado a la Asistente Social Juanita Aravena para incluirla en su memoria. Revista de Trabajo Social n.1. Santiago de Chile: Pontificia Universidad Católica de Chile. p. 44-45, 1970. SANDOVAl, A.; CASTILlO, D.; TRUjILlO, A.; CONCHA, M.A. Productividad de la mujer en el Asentamiento Alameda. Universidad de Chile: Temuco, 1972.

SUAREZ, M. Las empresas comunitarias campesinas en Chile. Instituto Interamericano de Ciencias Agrícolas de la Organización de Estados Americanos. Centro Interamericano de Desarrollo Rural y Reforma Agraria: Bogotá, 1972.

\section{Nota}

1 Cada entrevista fue realizada conforme a los protocolos establecidos en la Declaración de Helsinki (1975 revisada en 1983).

\section{Patricia Castañeda Meneses}

patricia.castaneda@uv.cl

Trabajadora Social y Licenciada en Trabajo Social en la Universidad de Valparaíso.

Doctora en Ciencias de la Educación en la Universidad de Barcelona.

Académica e Investigadora en Escuela de Trabajo Social en la Universidad de Valparaíso, Chile.

\section{Ana María Salamé Coulon}

ana.saIame@ufrontera.cl

Trabajadora Social. Pontificia Universidad Católica de Chile.

Doctora en Ciencias de la Educación en la Universidad de Barcelona.

Académica e Investigadora en Departamento de Trabajo Social en la Universidad de La Frontera.

\section{Universidad de Valparaíso}

Escuela de Trabajo Social

Avda. Colón 2128 Valparaíso, Chile

\section{Universidad de La Frontera}

Departamento de Trabajo Social

Avda. Francisco Salazar 01145 Temuco, Chile 